\title{
Limites de l'apport de la société civile
}

L'éducation dans les États fragiles

Limits to the contribution made by civil society. Education in fragile States

Límites de la aportación de la sociedad civil. La educación en los Estados frágiles

\section{Thomas Poirier}

\section{OpenEdition} Journals

Édition électronique

URL : http://journals.openedition.org/ries/2195

DOI : $10.4000 /$ ries. 2195

ISSN : 2261-4265

Éditeur

Centre international d'études pédagogiques

Édition imprimée

Date de publication : 1 décembre 2011

Pagination : 95-104

ISBN : 978-2-85420-593-0

ISSN : $1254-4590$

Référence électronique

Thomas Poirier, «Limites de l'apport de la société civile », Revue internationale d'éducation de Sèvres [En ligne], 58 | décembre 2011, mis en ligne le 01 décembre 2014, consulté le 14 novembre 2019. URL http://journals.openedition.org/ries/2195; DOI : 10.4000/ries.2195 


\section{Limites de l'apport de la société civile}

\section{L'éducation dans les États fragiles}

\section{Thomas Poirier}

Angola, Sierra Leone, Afghanistan, Birmanie, Haïti : ces pays géographiquement éloignés ont tous en commun d'être classés dans la catégorie des États dits "fragiles », c'est-à-dire défaillants dans divers domaines et à différents degrés. Pour la plupart d'entre eux, l'objectif de donner d'ici 2015 à tous les garçons et filles les moyens d'achever un cycle complet d'éducation primaire ne sera pas atteint. Les États fragiles concentrent en effet près du tiers des 67 millions d'enfants non scolarisés dans le monde (Unesco, 2010², 20112), et les disparités d'accès et d'offre en éducation y sont souvent plus aiguës que dans les autres pays en développement. Pays pauvres aux capacités institutionnelles faibles, les États fragiles sont généralement incapables de s'acquitter efficacement de leurs fonctions essentielles pour le développement (Chauvet et Collier, 2005) alors que la réalisation des objectifs de l'Éducation pour tous (EPT) apparaît comme un mécanisme fort de lutte contre la pauvreté et un facteur déterminant de la croissance économique. Le statut de l'éducation comme bien public mondial amène ainsi une partie des opinions internationales à admettre le droit à l'éducation des individus comme opposable à toute forme d'organisation sociale ou politique, en particulier dans les États qui ne s'engagent pas assez dans la lutte contre la pauvreté. Dans la mesure où la communauté internationale a consacré le partenariat comme moyen d'atteindre ces objectifs (Comhaire et Mrsic-Garac, 2007), parents, communautés et organisations de la société civile sont censés être étroitement associés à la conception des politiques d'éducation. Dans le cadre de l'EPT, la société civile peut être définie selon les critères retenus par l'Unesco, à savoir «l'ensemble des associations à caractère non gouvernemental et à but non lucratif travaillant dans le domaine de l'éducation. En font partie, entre autres, les ONG et les réseaux de campagne, les associations d'enseignants et les communautés religieuses, les associations communautaires et les réseaux de recherche, les associations de parents d'élève et les organismes professionnels, les associations d'étudiants ainsi que divers mouvements sociaux $»^{3}$. Ces organisations se caractérisent généralement par leur implantation au niveau local et certaines reçoivent le soutien d'ONG internationales (Save the Children, World Learning, etc.). L'une de leurs fonctions est alors de

1. Unesco, 2010. Atteindre les marginalisés. Rapport mondial de suivi sur l'Éducation pour tous. Unesco, Paris, 553 p. 2. Unesco, 2011. La crise cachée : les conflits armés et l'éducation. Rapport mondial de suivi sur l'Éducation pour tous. Unesco, Paris, $463 \mathrm{p}$.

3. http://www.unesco.org/education/efa/fr/partnership/civil_society.shtml (consulté le 22/10/2011). 
fournir une offre alternative d'éducation, là où l'action de l'État est inexistante ou insuffisante. Ce partage des responsabilités conduit à réduire le rôle centralisateur de l'État dans les politiques éducatives dont le pilotage, décentralisé, incomberait ainsi aux communautés locales. Cette gouvernance de l'éducation peut-elle alors se substituer à l'offre habituelle d'éducation par l'État? Dans le domaine de l'éducation, les fondements actuels de cette gouvernance mondiale amènent également à repenser l'architecture de la coopération internationale qu'il convient cependant de confronter aux terrains particuliers des États fragiles.

\section{L'ÉDUCATION DANS LES ÉTATS FRAGILES}

Pour une majorité d'États fragiles, principalement localisés en Afrique subsaharienne, l'objectif de la scolarisation primaire universelle fait figure de chimère. Malgré les efforts engagés par la communauté internationale depuis le Forum de Dakar en 2000, certains pays seront dans l'incapacité de scolariser tous les enfants d'ici 2015. En 2008, le taux net de scolarisation en Afrique subsaharienne atteignait $76 \%{ }^{4}$, soit 20 points de plus qu'en 2000 (58\%). Si cette évolution indique une plus grande participation, près du tiers de l'ensemble des pays d'Afrique subsaharienne affichait un taux inférieur à la moyenne continentale. Ainsi, en Erythrée, ce taux n'atteint pas les $40 \%$ tandis qu'au Niger, ce taux franchit à peine la barre des $50 \%$. D'autres pays, considérés comme fragiles, ont cependant déjà réalisé la scolarisation primaire universelle ou sont en voie de l'atteindre. C'est le cas notamment du Zimbabwe (90\%) ou du Rwanda (96\%) et dans une moindre mesure du Kenya ( $82 \%)$. En termes d'efficacité interne des systèmes éducatifs, de nombreuses disparités entre pays peuvent également être observées. En 2007, le taux d'abandon (toutes années confondues) était de $10 \%$ en Côte d'Ivoire, de $30 \%$ au Congo, de $45 \%$ en Guinée et de $70 \%$ au Tchad. En Afrique subsaharienne, ce taux culminait en moyenne à $30 \%$, soit moins de quatre points par rapport à 2003 (33,9\%). L'un des indicateurs les plus préoccupants en regard des objectifs de l'EPT concerne le taux de survie en cinquième année du primaire. Les données disponibles montrent qu'entre 1999 et 2007, parmi les États fragiles d'Afrique subsaharienne, la Côte d'Ivoire est le seul pays où ce taux a significativement augmenté, passant de $62 \%$ à $90 \%$. Sur la même période, ce taux a diminué dans les autres pays fragiles : de $58 \%$ à $46 \%$ au Lesotho, de $51 \%$ à $40 \%$ en Éthiopie et de $93 \%$ à $73 \%$ en Érythrée. Pour l'ensemble des pays d'Afrique subsaharienne, ce taux accuse en moyenne une diminution de près de trois points, s'établissant à $70 \%$ en 2007 contre $72,6 \%$ en 2003. Cette tendance, illustrée par le graphique ci-contre, confirme les difficultés de rétention des élèves dans les États fragiles : ceux-ci s'inscrivent certes davantage qu'il y a dix ans dans les établissements scolaires mais sont moins nombreux à y achever le cycle primaire.

4. Données publiées en 2011 pour l'année 2008 par l'Institut de statistique de l'Unesco (ISU). 
Taux d'achèvement total du primaire en Afrique subsaharienne

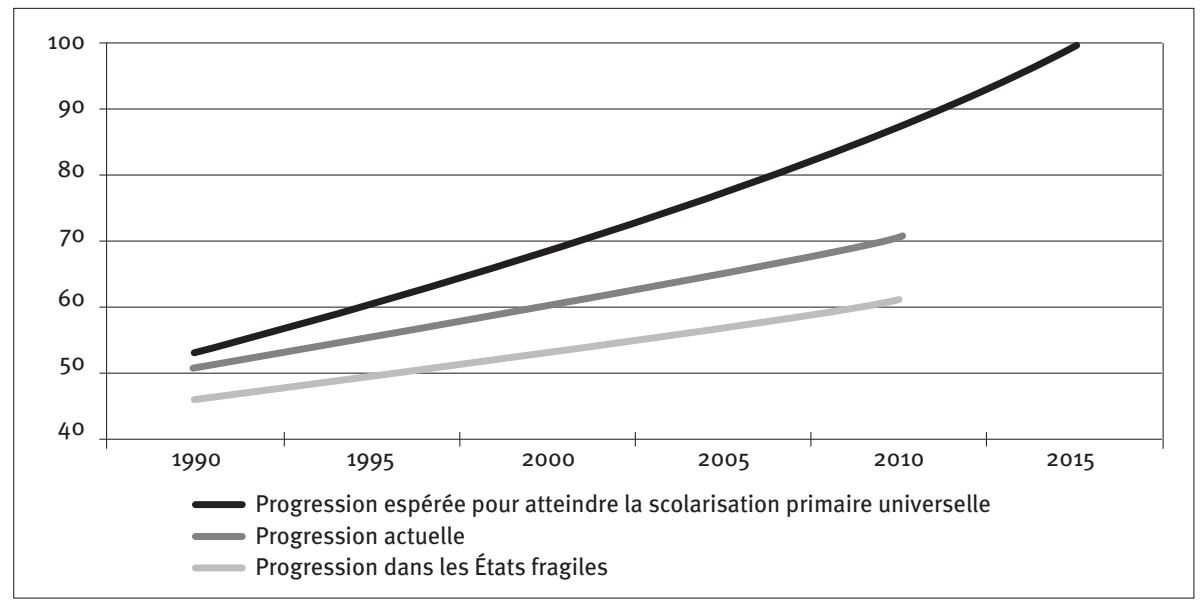

Source : Banque mondiale 5 .

Dans le cadre des objectifs de l'EPT, le financement des systèmes éducatifs reste une question centrale. Les pays d'Afrique subsaharienne sont ceux qui investissent le plus dans le primaire, en rapport aux dépenses globales d'éducation (46\% en moyenne, soit douze points de plus que la moyenne mondiale). Ces dépenses se révèlent néanmoins insuffisantes et selon les principes adoptés en 2000, l'aide extérieure doit permettre de combler les déficits de financement. Si l'aide a doublé depuis 2002 pour atteindre aujourd'hui 4,7 milliards US\$, elle est cependant encore loin des 16 milliards US\$ estimés nécessaires (Unesco, 2011, op. cit.). L'aide à l'éducation de base stagne en effet depuis 2007 et en Afrique subsaharienne, elle a diminué d'environ $6 \%$ par enfant en âge de fréquenter l'école (op. cit.). Certains États fragiles concentrent également cette aide internationale. Ainsi, en moyenne, sur la période 2007-2008, l'Afghanistan, l'Irak et le Pakistan ont reçu à eux trois plus d'aide à l'éducation de base que tous les pays fragiles d'Afrique subsaharienne réunis ${ }^{6}$ (382 contre 314 milliards US\$), une tendance inquiétante qui confère à certains pays, comme la République centrafricaine, le statut d' " orphelin de l'aide ».

Il existe aujourd'hui un consensus à l'échelle internationale pour dire que des États sont en situation de fragilité. Celle-ci s'évalue avant tout négativement, à partir de deux critères fondamentaux : la faiblesse des performances économiques et l'impuissance effective de la puissance publique (Châtaigner et Gaulme, 2005), auxquelles il convient d'ajouter une instabilité sociopolitique

5. http://ddp-ext.worldbank.org/ext/GMIS/gdmis.do?siteld=2\&amp;menuld=LNAVo1REGSUB6 (consulté le 15/07/2011).

6. En 2007, le Country Policy and Institutional Assessment (CPIA) de la Banque mondiale a recensé 34 États fragiles, dont 21 en Afrique subsaharienne. 
chronique. Un échec des Objectifs du millénaire pour le développement dans ces pays, particulièrement ceux de l'EPT, serait également considéré comme un échec de la communauté internationale. Cette perspective, conjuguée à une perception grandissante des États fragiles comme une menace pour la sécurité internationale, sont les principales raisons qui ont amené les bailleurs et organisations internationales à intensifier leur réflexion sur ces pays.

\section{UNE GOUVERNANCE DE L’ÉDUCATION DÉCENTRALISÉE}

\section{La société civile au cœur du dispositif}

Dans son rapport 2009 sur l'EPT ${ }^{7}$, l'Unesco souligne qu' « une bonne gouvernance pourrait aider à renforcer la responsabilité, accroître la participation et réduire les inégalités dans l'éducation ». Des recommandations pour une gouvernance de l'éducation plus efficace ont notamment été publiées dans deux rapports, le premier par la Banque mondiale en $2003^{8}$, le second par les Nations Unies en $2005^{9}$. Cette réforme de la gouvernance comprend ainsi :

l'éducation ;

- la décentralisation du financement et du "management» de

- l'implication des parents dans le «management» des écoles de base ;

- l'introduction de choix mécaniques;

- l'expansion des ONG et la fourniture de service public-privé pour stimuler la concurrence.

Dans le cadre de ce paradigme participatif, la société civile, principalement à travers les associations de parents d'élèves, des communautés ou d'ONG locales, est amenée à jouer un rôle accru dans la prise de décision, notamment en cas de défaillance de l'État à fournir le bien «éducation». L'implication de la société civile permettrait à la fois l'appropriation nécessaire à toute perspective à long terme (les objectifs de l'EPT), la transparence, la prise en compte des intérêts des plus pauvres et donc la réduction de la pauvreté et des inégalités d'accès aux services publics (Comhaire et Mrsic-Garac, op. cit.). Afin que le partenariat entre les différents acteurs (État, familles, communautés, ONG) engendre les effets positifs attendus, une série de conditions préalables est nécessaire. Les organisations de la société civile devraient être représentatives, légitimes, autonomes et maîtriser les enjeux relatifs à leur domaine d'activité. Les

7. Unesco, 2009. Vaincre l'inégalité. L'importance de la gouvernance. Rapport mondial de suivi de l'Éducation Pour Tous. Unesco, Paris, $505 \mathrm{p}$.

8. World Development Report 2004: making services work for poor people. World Bank and Oxford University Press, $288 \mathrm{p}$.

9. Task Force on Education and Gender Equality: Toward Universal Primary Education: Investments, Incentives and Institutions. UN Millenium Project, Earthscan, Sterling, 2005, 207 p. 
gouvernements devraient être engagés dans la lutte contre la pauvreté, partager leur pouvoir de décision avec leurs partenaires et avoir les capacités institutionnelles et techniques pour assurer un réel processus participatif. Les bailleurs devraient de leur côté respecter les priorités du gouvernement (op. cit.).

En quelques années, la fonction de ces organisations de la société civile dans les politiques de développement est passée d'une approche technique ("exécutant») à une approche politique, en ce sens que la société civile est censée pouvoir agir sur les politiques et les normes. La forme préconisée par la plupart des organisations internationales serait en soi une organisation éducative centrée sur la société civile, où le rôle de l'État serait d'émettre des normes et programmes sur le système éducatif. L'éducation serait vue comme décentralisée, pilotée localement par la société civile, dans un souci d'"empowerment».

Or, dans les contextes forts variés des États fragiles, cette approche unique soulève de nombreuses interrogations : la participation est-elle vraiment précieuse et pertinente, à n'importe quel stade, à n'importe quel endroit et avec n'importe qui ? Dans les États fragiles, plus qu'ailleurs, une condition délicate vient de l'interdépendance des domaines politique, économique, social et de celui de la sécurité : l'échec de l'un d'eux risque d'entraîner l'échec de tous les autres $(\mathrm{OCDE}, 2006)^{10}$. Ces paramètres sont autant d'obstacles à la mise en place d'une gouvernance locale de l'éducation. Une offre efficace d'éducation suppose aussi une organisation démocratique de la vie politique locale. Bien souvent, les domaines d'action entre les autorités locales élues et les formes d'organisation de la société civile (associations de parents d'élèves, comités scolaires) ne sont pas clairs et peuvent engendrer des tensions (Mundy, 2008).

Le contexte spécifique des États fragiles rend ainsi la participation de la société civile beaucoup plus aléatoire, les conditions n'étant souvent pas réunies pour que celle-ci puisse y jouer un rôle déterminant, et la communauté internationale voit souvent la société civile comme un bloc homogène qui aurait les mêmes caractéristiques partout. La prescription d'une bonne gouvernance universelle, fondée sur une participation accrue des citoyens, se heurte ainsi aux spécificités locales, spécialement dans les États fragiles, où le risque d'instabilité sociopolitique rend tout projet incertain. De plus, des études empiriques font encore défaut pour démontrer les avantages de la participation des organisations de la société civile. L'enthousiasme suscité par ces dernières semble en effet s'appuyer essentiellement sur les échecs des politiques de coopération antérieures et sur les espérances placées en elles (Rombouts, 2006).

La participation des organisations de la société civile est désormais abordée comme un processus politique. Puisque l'État ne parvient pas à combler la demande, il est contraint d'abandonner sa fonction régalienne d'éducation au profit d'une hypothétique société civile. Pourtant, la question d'une régulation

10. Les approches à l'échelle de l'ensemble de l'administration pour les États fragiles, $62 \mathrm{p}$. 
publique se pose, afin notamment de garantir le fonctionnement d'un système éducatif selon des normes légales. Plus largement, dans le contexte des États fragiles, restaurer une puissance publique forte pourrait permettre aux populations de redonner confiance à un État de droit souvent déconsidéré pour son ineptie ou pour sa corruption.

\section{Le rôle réduit de l'État}

La décentralisation de la gouvernance de l'éducation a pour effet que le système éducatif devient localement contrôlé par de petites unités privées (ONG locales d'aide au développement en éducation, associations de parents d'élève), le rôle de l'État n'étant plus que d'émettre des signaux et de fixer des normes et des programmes. Si la décentralisation peut améliorer une éducation publique, certaines conditions analysées plus haut doivent cependant être réunies, ce qui est loin d'être le cas dans les États fragiles. Une décentralisation "forcée » pourrait conduire à des effets contre-productifs et au contraire détériorer davantage une situation déjà fragile (Altinok, 2004). L’agenda international a néanmoins fixé la décentralisation comme une des clés de la "bonne gouvernance », avec l'idée que les bureaucraties centralisées ne sont pas les fournisseurs les plus équitables de services éducatifs. Dans un rapport publié en $2006^{11}$, la Banque mondiale indique que "le financement public cultive des grandes machines bureaucratiques et des groupes d'intérêt forts, dont les pressions pourraient aboutir à l'inertie ". S'il faut en effet souligner que les administrations, dans la plupart des États fragiles, outre leurs faiblesses, sont souvent minées par la corruption, l'alternative à l'offre de services publics par l'État pose la question des capacités de la société civile et de ses organisations à fournir le bien "éducation ", notamment aux plus pauvres.

La légitimité des ONG ou des organisations locales de la société civile peut également être sujette à caution. Celles-ci n'ont par exemple pas à se soucier d'élections futures et ne sont pas redevables à l'électorat. Au niveau local, le caractère de certaines ONG (religieuses notamment) amène aussi à questionner leur motivation, d'autant plus que l'évaluation de leurs activités reste encore faible et que, dans certains cas, elles agissent de façon opaque et en dehors de tout contrôle (Besley et Ghatak, 2008). À la différence de la plupart des ONG internationales qui ont pignon sur rue et dont les actions font l'objet d'un suivi approfondi de la part des bailleurs de fonds, les organisations locales de la société civile souffrent généralement d'un manque de visibilité, de l'absence de lisibilité de leurs activités sur le terrain et d'un manque de spécialisation (Cossu, 2004). Il existe également un risque de détournement des ONG à des fins de rentes aux dépens des donateurs. Comme le souligne Poncelet et Pirotte (2007), « bien des 
organisations de la société civile autoproclamées et financées comme telles ne sont que des petites entreprises ou encore des agences publiques déguisées ». C'est le cas notamment au Libéria. Dans un entretien publié en $2007^{12}$, le ministre de la Planification et des affaires économiques de l'époque, Toga McIntoch, explique que sur les 96 ONG internationales et 700 ONG locales implantées dans son pays, certaines ont été créées uniquement pour accéder à un financement : "si quelqu'un veut travailler dans le secteur privé, il se nomme lui-même ONG ». On assiste alors à une utilisation des ONG à des fins lucratives, dans un contexte où les perspectives d'emplois, notamment dans le secteur formel, sont souvent limitées. D'une part, le secteur privé fait face à plusieurs contraintes qui sont autant de freins à son développement: quasi-absence d'un État de droit, une régulation inappropriée voire absente, lourdeur bureaucratique, insécurité, corruption, etc. (Sang, 2007). D'autre part, depuis les politiques d'ajustement structurel, la fonction publique ne se présente plus comme un débouché «naturel» pour les diplômés. Une situation d'autant plus difficile que la crise économique touche également les États fragiles où «les destructions d'emplois et la raréfaction des débouchés ont touché des millions de ménages » selon un rapport du Fonds monétaire international publié en $2010^{13}$.

Enfin, il convient de souligner le paradoxe qui voit une communauté internationale plaider d'un côté pour des États forts, capables d'assurer la sécurité (intérieure et régionale) et de l'autre, appeler à un désengagement de ces derniers dans des domaines symbolisant pourtant le retour d'une puissance publique effective. De nombreux travaux réalisés ces dernières années soulignent l'importance de la consolidation de l'État et du renforcement de ses institutions pour favoriser le développement économique et social notamment. Ainsi, lorsqu'un gouvernement donne la priorité au secteur social, il envoie à la population le signal d'un retour durable de la stabilité, ce qui a pour effet, notamment, d'augmenter la confiance en la paix, nécessaire aux investissements (Collier et Hoeffler, 2002).

Dans la mesure où certains pays se montrent incapables de répondre aux attentes fixées par l'agenda international, la société civile est amenée à jouer un rôle accru pour réduire les inégalités d'accès, palliant certes les déficiences de l'État mais empiétant également sur des fonctions traditionnellement régaliennes. L'approche participative, développée dans le cadre des Objectifs du millénaire pour le développement, soulève aussi de nombreuses interrogations, notamment sur les modalités renouvelées de l'intervention internationale. Élevée au rang de bien public mondial, l'éducation devient un droit opposable. D'aucuns y verront alors une forme de légitimité pour franchir le pas vers le devoir d'ingérence, dès lors que les populations sont entravées dans leur droit.

12. In Capacity.org $n^{\circ}$ 32, Décembre 2007.

13. Fonds monétaire international, 2010. Perspectives économiques régionales : Afrique subsaharienne. Washington, D.C., 113 p. 


\section{Aide internationale : un devoir d'ingérence éducatif?}

L'éducation comme bien public mondial s'inscrit dans un contexte de mondialisation des marchés et de limites des interventions des États. Il est désormais largement admis que certains secteurs, comme l'éducation, revêtent un caractère public au niveau international et qu'en fournir l'accès, notamment aux plus pauvres, dépasse le cadre défini des frontières nationales. À ce titre, le bien « éducation » est considéré comme public, d'une part parce que sa production résulte de choix collectifs, d'autre part du fait des externalités qu'il engendre sur l'ensemble des acteurs de la société. L'offre efficace de biens publics constitue ainsi un volet important de la stratégie de réduction de la pauvreté.

Adoptant une position normative des biens publics mondiaux, les théories néoclassiques soulignent que l'État se doit d'intervenir uniquement pour répondre aux imperfections du marché, tout en limitant a priori les fonctions qu'il doit assumer (Gabas et Hugon, 2001). Ainsi, la définition économique des biens publics mondiaux, inscrite dans une perspective libérale, se réfère aux défaillances des marchés et aux moyens d'y remédier par des arrangements collectifs, en donnant une dimension internationale aux politiques sectorielles nationales. Une autre conception, celle de l'économie politique mondiale, considère que ce sont les acteurs qui définissent l'intérêt général et non les forces du marché. Cette vision pose donc la question des procédures et des décisions permettant de hiérarchiser les biens publics, de les produire et de les financer par la mise en place d'encadrements normatifs (Bissiriou et Kern, 2005). En privilégiant les patrimoines communs, lesquels dépendent des choix collectifs, l'économie politique tend à concevoir l'éducation comme un bien public mondial dont les caractéristiques ne sont pas figées, mais évolutives selon des contextes en proie à de profondes mutations (op. cit.). Il apparaît donc nécessaire de fonder les biens publics sur des compromis et des intérêts supérieurs à définir lors de négociations internationales.

Transposée à l'échelle internationale, la théorie des biens publics pointe la défaillance à la fois des marchés et des États à produire ces biens publics. Suivant la logique d'économie politique mondiale, ce ne serait alors plus aux seuls États de produire le bien "éducation", mais aussi aux instances internationales, pour des raisons de solidarité internationale mais aussi au nom d'un patrimoine commun. Les enjeux liés à l'éducation comme droit opposable dépassent ainsi le cadre des frontières nationales. Une régulation internationale de l'éducation nécessite alors un transfert des souverainetés, suivant un principe de subsidiarité, et justifie pour certains un droit d'ingérence, dans la mesure où certains pays ne respectent pas les règles d'une coopération efficace inscrite dans le cadre de la bonne gouvernance. Pour autant, la production centralisée d'éducation au niveau mondial, contrôlée par une instance multilatérale ayant 
le pouvoir légitime de veiller au respect des règles collectivement élaborées, a pour l'instant ses limites, notamment en matière de financement. La question du bien public "éducation » renvoie donc à une souveraineté des citoyens faisant des choix collectifs. En ce sens, l'approche participative à la coopération peut adopter une attitude de contournement des États non coopératifs. Elle a ainsi élargi de manière substantielle le rôle de la société civile pour atteindre les objectifs de la scolarisation primaire universelle.

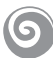

L'échec probable de certains pays les plus fragiles dans la réalisation de la scolarisation primaire universelle a placé la communauté internationale face à un défi majeur. En privilégiant une approche participative, centrée sur la société civile, la stratégie pour atteindre les objectifs de l'EPT suscite des interrogations, tant dans sa conception que dans sa mise en œuvre. Les organisations internationales semblent parfois céder à la tentation de voir dans la participation de la société civile un processus démocratique de gestion de l'offre scolaire, permettant de se substituer à un État incapable de fournir une éducation de base à la population. Certains y voient aussi un devoir d'ingérence, dans la mesure où l'éducation est perçue comme un droit opposable.

À travers les associations de parents d'élèves, des communautés ou d'ONG locales, l'action de la société civile sur l'offre d'éducation semble encore limitée par un manque à la fois de légitimité et de capacité (Comhaire et Mrsic-Garac, Besley et Ghatak, op. cit.). D’autant que sur le terrain spécifique des États fragiles, les populations locales peuvent ne pas adhérer à une offre d'éducation dans laquelle elles ne voient pas d'intérêt à investir (Pritchett, 2001). Les normes de bonne gouvernance, liées à la pression d'achèvement de l'EPT, amènent ainsi à s'interroger sur les structures organisationnelles et de financement qui sont en mesure de fournir efficacement le bien "éducation ». Dans les contextes particuliers des États fragiles, il convient de renforcer la réflexion autour du rôle dévolu à la puissance publique. Les possibilités de retour à un État de droit, légitimé, effectif et capable d'assurer la sécurité, sont des conditions nécessaires pour permettre la reconstruction d'un système éducatif dans ces pays. Brinkerhoff (2005) distingue trois grandes étapes interdépendantes permettant de reconstruire une gouvernance dans les États fragiles : recouvrer une légitimité (reconstituting legitimacy); rétablir la sécurité (re-establishing security); reconstruire une effectivité (rebuilding effectiveness). Cependant, le caractère spécifique de ces pays conduit certainement à relativiser l'agenda international et à revoir les objectifs qu'ils ne seront pas à même de tenir, compte tenu de leurs difficultés à sortir de la fragilité. 


\section{BibLIOGRAPHIE}

ALTINOK N. (2004) : La Banque mondiale et l'éducation en Afrique subsaharienne. De grandes paroles pour de petites actions ? Les Cahiers de l'IREDU n 64, 226 p.

BESLEY T., GHATAK M. (2008) : L'offre de services publics par les acteurs non gouvernementaux. Revue d'économie du développement 4(22): 89-108.

BISSIRIOU G., KERN F. (2005) : L'éducation comme bien public mondial est-elle compatible avec l'Accord général sur le commerce et les services? Mondes en Développement 4(132): 39-55.

BRINKERHOFF D. W. (2005) : Rebuilding governance in failed states and postconflict societies: core concepts and cross-cutting themes. Public administration and development 25(1): 3-14.

CHÂTAIGNER J.M., GAULME F. (2005) : Agir en faveur des acteurs et des sociétés fragiles. Document de travail n ${ }^{\circ}$, Agence Française de Développement, 25 p.

CHAUVET L., COLLIER P. (2005): Development effectiveness in fragile states: spillovers and turnarounds. Centre for the Study of African Economies, Department of Economics, Oxford University, 45 p.

COLLIER P., HOEFFLER A. (2002) : Aid, policy, and growth in post-conflict ocieties. Policy research working paper 2902, World Bank, Washington, D.C., 24 p.

COMHAIRE G., MRSIC-GARAC S. (2007) : La «participation » des parents dans des contextes de systèmes éducatifs en crise. Études de cas au Bénin et en République Démocratique du Congo. Mondes en développement 3(139): 43-56.

COSSU M. (2004) : La contribution des OSC à l'éducation de base. In Les organisations de la société civile et la lutte contre la pauvreté en Afrique subsaharienne, actes du séminaire de recherche de Cotonou du GRAP OSC, p. 50-59.

GABAS J.J., HUGON P. (2001) : Les biens publics mondiaux et la coopération internationale, L'Économie Politique 4(12): 12-31.

MUNDY K. (2008) : Civil society and its role in the achievement and governance of "Education for all». Paper commissioned for the EFA Global Monitoring Report 2009: Overcoming inequality: why governance matters. Unesco, Paris, 32 p.

PONCELET M., PIROTTE G. (2007) : L'invention africaine des sociétés civiles : déni théorique, figure imposée, prolifération empirique. Mondes en développement 3(139): 9-23.

PRITCHETT L. (2001): Where has all the education gone? World Bank Economic Review 15(3): 367-391.

ROMBOUTS H. (2006) : Civil society participation in fragile states: critical thoughts on the new development paradigm and its implementation. Discussion paper, Institute of development policy and management, University of Antwerp, $48 \mathrm{p}$.

SANG M. (2007) : Le secteur privé : une réponse aux insuffisances de l'État. In CHATAIGNER J.-C., MAGRO H., 2005. États et sociétés fragiles. Entre conflits, reconstruction et développement. Paris, Karthala, 565 p. 